\title{
ESSAY REVIEW
}

\section{Evidence-Based Analysis of the COVID Pandemic}

COVID: Why Most of What You Know is Wrong by Sebastian Rushworth. Karneval Publishing, 2021. 130 pp. \$18, paper, \$9.50 Kindle. ISBN-13: 978-91-88729-83-5.

\section{Reviewed by Henry H. Bauer}

hhbauer@vt.edu

www.henryhbauer.homestead.com

https://doi.org/10.31275/20212157

Creative Commons License CC-BY-NC

This short book suggests plausible answers to much of what has seemed inexplicable or unbelievable about what governments, the World Health Organization, and popular media have disseminated about the officially declared global pandemic.

The author is a recently graduated Swedish physician who recognized even during his training that many of the generally accepted shibboleths about medical matters are not evidence-based; are often, in fact, contrary to the available evidence.

Irrespective of his suggestions about COVID, several points in this book are important for everyone to know:

1. Modern medicine focuses on the handling of emergencies but says "extremely little about how to avoid chronic disease and maximize long term health" (p. 7).

2. More than half of the widely accepted recommendations about nutrition are nonsense, without any basis in solid evidence ( $p$. 8): to eat more fruit, fish, vegetables, whole-grain cereals, and less salt, saturated fat, or meat.

3. Much purportedly scientific medical information stems from inappropriate use of surrogate endpoints and improper statistical analyses (pp. 34-39): The usual criterion for statistical 
significance $(p \leq .05)$ is arbitrary and very weak. The typical marketing ploy of citing relative rather than absolute risks is highly misleading (pp. 40-46).

How deadly is COVID?

The salient point is that deaths associated with COVID have affected primarily the elderly.

In Sweden, the average age of those dying from COVID was 84; while overall the average age at death (from all causes) in Sweden was 82 (p. 51). This supports the view that COVID, like so many other infections, is particularly dangerous for people with co-morbidities; thus it would be more accurate to talk of deaths with COVID than from COVID. This point is underscored by a cited study from 2017 which reported that frail elderly hospitalized people died more frequently after suffering "the common cold" (rhinoviruses) than after infection by actual influenza (p. 49)!

The suggestion is, in other words, that deaths with COVID have, in the main, not added an appreciable number of excess deaths, but rather replaced deaths that would otherwise have been attributed to different causes. That suggestion seems strongly supported by the cited data that the overall mortality in Sweden in 2020 was just $5 \%$ larger than the average for the previous five years (p. 41), balancing statistically the $5.7 \%$ lower overall mortality for the previous five years in 2019 (p. 93).

However, data reported by EUROMOMO ${ }^{1}$ do show substantial excess deaths for a number of countries, as does the Centers for Disease Control \& Prevention for the USA ${ }^{2}$ with excess deaths in 2020-2021 considerably more than in the severe "flu" season of 2018 . More data are surely needed before we can be quite certain.

Rushworth cites the overall infection fatality rate (IFR) of COVID as $0.15-0.2 \%$, but only half that for people younger than 70 . By comparison, the 1918 flu pandemic had an estimated rate of $2.5 \%$ and was highly dangerous for young people. However, the death rate is not the only way to measure a society's burden of disease; the average age of death makes it possible also to calculate the overall number of years of life lost. From that point of view, the social burden of COVID may be no greater than the burden imposed by a lack of childhood vaccinations for measles, polio, and other common infectious diseases that are avoidable by vaccination (pp. 50-54). 


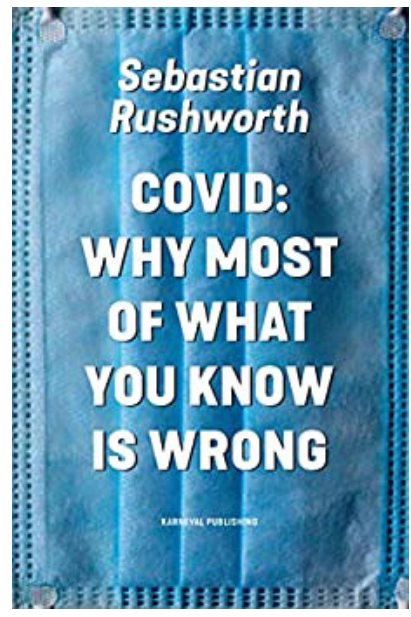

Rushworth suggests also that socalled "long COVID"—debilitating symptoms continuing long after the acute illness is past-may not be a distinct new entity. It is similar to what is experienced by people who have suffered an episode of any serious illness with intensive care: Some $60 \%$ of people treated in intensive care for any condition still show some cognitive impairment a year later (p. 55). An MRI study of people claiming "long COVID" symptoms did turn up quite a lot of indications of impaired function by various organs, but the lack of a control group renders this quite meaningless (pp. 6o-61): A random sample of symptom-free individuals, particularly older ones, would also show all sorts of minor, unimportant deterioration in a number of organs.

The book has an excellent discussion of what COVID tests are and what they can show; and how very misleading the promiscuously disseminated data can be as a result of false-positive and false-negative test results (pp. 62-73). Everyone should read this, over and over until it is fully digested, to appreciate the (possible lack of) significance of the sensitivity and specificity of a test-any test, not merely for COVID. Few untutored people know that the significance of test results, the proportion of false-positives and false-negatives, depends not only on the stated sensitivity and specificity of the test but also on the prevalence in the population of what is being tested for. One consequence is that public policies based simply on the number of apparently positive tests could well be misplaced, seeming to indicate that the pandemic continues when in reality it may be already over (p. 70).

Have lockdowns prevented COVID deaths? Rushworth quite properly points out that the widely accepted belief in the efficacy of lockdowns rests on two highly unreliable sources: official statements from China, and statistical modeling, bearing in mind that modeling should never be regarded as sound evidence (pp. 74-75).

Nevertheless, Rushworth concludes that lockdown seems ineffective (p. 88), does not reduce mortality caused by COVID, even that "nothing that various world governments have done to combat 
COVID seems to have had any effect whatsoever on the number of deaths" (p. 78).

That conclusion seems less than convincing, however, for several reasons:

1. The data come from only the first few months of the pandemic.

2. Some of the cited studies relied on inappropriate statistical analyses (pp. 77, 79).

3. Perhaps the best-cited study indicated that COVID is "unbelievably infective" (p. 86) through immediate personal contact but that it does not spread readily in or from spaces recently occupied by infected people; and that most infected individuals are not very infectious while a few seem to be "super spreaders" (pp. 83-88).

The conclusion that facemasks do little to stop the spread of COVID seems unconvincing for the same reasons, and also because the cited studies were of the spreading of respiratory infections in general, not of COVID itself (those studies showed a small effect only of facemasks, with $\mathrm{N}_{95}$ masks much better than surgical masks let alone cloth masks).

Since COVID is unusually infectious (p. 86), something that might have only a small impact on the spreading of cold or flu viruses might well have a more appreciable impact with COVID. Indeed, the best-controlled cited study (pp. 83-88) showed that strict isolation, distancing, and mask-wearing, together with testing and contact tracing and quarantining, essentially prevented spreading of infection from one platoon of Marine recruits to recruits in other platoons that used the same spaces at different times. Lockdown is surely an extreme case of quarantining, since it ensures the isolation of any super-spreaders and does not require voluntary adherence to guidelines or even mandates for mitigation methods. Anecdotal media reports comparing the experiences of different countries seem to suggest that lockdowns have been somewhat effective; either that, or very early testing, contact tracing, and quarantining seem the only two possible reasons why such countries as Taiwan or New Zealand have suffered almost not at all compared to, say, Britain, Brazil, or the United States.

What else, if anything, reduces risks from COVID? The worst outcomes seem to correlate with obesity, age, and low income (p. 77). 
Vitamin D appears to reduce risk, a possible explanation for the greater impact of COVID on dark-skinned people, whose complexion is a barrier to the body's generation of vitamin D by sunlight (p. 91).

As to the safety and effectiveness of the vaccines, the book is equivocal, for well-explained reasons (pp. 112-131).

Rushworth makes a strong case that the virus may be no more "deadly" than other severe respiratory viruses. Nevertheless, COVID generated a disproportionate degree of global hysteria. This book ascribes the highly damaging impact of the disease not to inherent properties of the virus but to misdeeds and dysfunctions in how societies responded. These speculations (pp. 132-316) I find very plausible, indeed at least tentatively convincing.

Not discussed is the possibility that COVID originated in a laboratory, possibly involved in experiments relevant to biological warfare. In that case, it might have entirely un precedented characteristics.

Also not discussed is that this pandemic surely demonstrates that "ordinary flu" seasons are not handled particularly well. Perhaps, for example, elders, say 65 or older, might be advised during "normal flu seasons" to practice judicious distancing and wearing of N95-masks in public, which would likely prevent more illness than the usual "flu shot."

I recommend this book without reservation as a reliable analysis, based admittedly on only the first months of the epidemic. Endnotes give links to significant cited data. There is no index, but that is not particularly troubling since the chapters are quite short (12 pages long on average) and accurately described by their titles.

\section{NOTES}

1 EUROMOMO.

https://www.euromomo.eu/about-us/history/) https://www.euromomo.eu/graphs-and-maps\#excess-mortality

${ }^{2}$ Centers for Disease Control \& Prevention; weekly number of deaths (from all causes)

https://www.cdc.gov/nchs/nvss/vsrr/covid19/excess_deaths.htm Graph is about halfway down the webpage. 